\title{
New Topology Control base on Ant Colony Algorithm in Optimization of Wireless Sensor Network
}

\author{
Zana Azeez Kakarash $^{1 *}$, Sarkhel H.Taher Karim ${ }^{2}$, Nawroz Fadhil Ahmed ${ }^{3}$, Govar Abubakr Omar ${ }^{4}$ \\ ${ }^{1 \& 3}$ Department of Engineering, Faculty of Engineering and Computer Science, Qaiwan International University, Sulaymaniyah, Kurdistan Region, \\ Iraq; Department of Information Technology, Kurdistan Technical Institute, Sulaymaniyah, Kurdistan Region, Iraq \\ ${ }^{2}$ Department of Computer Science, College of Science, University of Halabja, Halabja, Kurdistan Region, Iraq \\ ${ }^{4}$ Department of Information Technology, College of Science and Technology, University of Human Development, Sulaymaniyah, Kurdistan Region \\ Iraq
}

\begin{abstract}
Received 18 January 2021; revised 24 March 2021;
accepted 30 March 2021; available online 14 April 2021
\end{abstract}

doi: $10.24271 /$ psr.22

\begin{abstract}
Wireless sensor networks (WSNs) have found great appeal and popularity among researchers, especially in the field of monitoring and surveillance tasks. However, it has become a challenging issue due to the need to balance different optimization criteria such as power consumption, packet loss rate, and network lifetime, and coverage. The novelty of this research discusses the applications, structures, challenges, and issues we face in designing WSNs. And proposed new Topology control mechanisms it will focus more on building a reliable and energy efficient network topology step by step through defining available amount of energy for each node within its cluster, sorting all within header, and selecting an active one (more power header) for signal routing. While sensor cover topology demonstrates network monitoring capability, connection topology should remain as a requirement for the successful delivery of information including queries, data collected, and control messages. How to build an optimized coating topology while remaining efficient and low-cost connection is not well understood and needs further research. Power control and power management are two different types of topology controllers. Also in our study, we examine network lifetime, compared to other schemas time of death of the first node and the last node, and found that network lifetime was increased. Finally, a topology control method for extending network lifetime is presented.
\end{abstract}

(C) 2021 Production by the University of Garmian. This is an open access article under the LICENSE

https://creativecommons.org/licenses/by-nc/4.0/

Keywords: Topology control, Wireless sensor network, Network lifetime, Energy, Ant colony algorithm.

\section{Introduction}

The history of sensor networks goes back to the Cold War era (mid-1950s) and the SOSUS voice surveillance system. The system was deployed by the United States to detect and track Soviet submarines in the North Pacific. The network is an extensive network of hydrophones connected by cable and covering the ocean environment. The system is currently being used by the NOAA National Institute to monitor current phenomena on the ocean floor ${ }^{[1]}$. Later on networks mainly consists of one core switch that provides a very high data transfer through connecting all the collected positions by a variety of cable media to the entire network switches which are installed in each location located in the network ${ }^{[2,3]}$. Recent advances in the field of wireless telecommunications have led to the design and

* Corresponding author

E-mail address: zana.azeez.k@gmail.com (Instructor).

Peer-reviewed under the responsibility of the University of Garmian. manufacture of sensors with low power consumption, small size, reasonable price and different applications. This technology created wireless sensor networks. WSNs provide reliable operations in various application areas including environmental monitoring, health monitoring, vehicle tracking system, military surveillance and earthquake observation ${ }^{[4,5,6,7]}$.

Wireless sensor networks are a distributed structure network consisting of a number of nodes with limited processing power as a sensor and one or more nodes that have different names depending on the network usage and their connection is usually wireless. The development of wireless communications and the production of small and varied parts of the operation have led to the increasing use of such networks ${ }^{[2,8,9,10,11]}$.

The nodes used in wireless sensor networks have other limitations in terms of power supply and lifetime in addition to the limited processing power, so the network lifetime will largely depend on the lifetime of the nodes used in the network ${ }^{[12,13,14]}$. The largest consumer of energy in wireless sensor networks is the sending and receiving of information. In addition to sending and receiving information, it is also worth noting that wireless sensor network 
nodes usually have different operating modes, which can switch from one state to another or long into one state ${ }^{[15,16]}$.

There are a large variety of routing protocols for WSNs, as well as classical routing and intelligent routing. ant colony optimization (ACO) ${ }^{[36]}$ may be a well-known intelligent algorithmic rule wherever complicated collective behavior emerges from the behavior of ants. collectively of the most successful swarm intelligence algorithms [37], it's additionally succeeded in some_elds of the planning of WSNs, like activity scheduling, node readying, target tracking, knowledge aggregation, and attack detection ${ }^{[38,39,40]}$. There additionally exist some ACO primarily based routing protocols in WSNs, like that in ${ }^{[41,42]}$. In ACO-based routing protocols, typically a colony of artificial ants travels through the network and appearance for ways between supply nodes and a destination node, as shown in Fig. 1. every ant chooses the relay node in step with a probability with relevance pheromone path and heuristic information. once an ant reaches the destination node, it travels backwards trough the path made and updates the pheromone trail ${ }^{[43]}$.

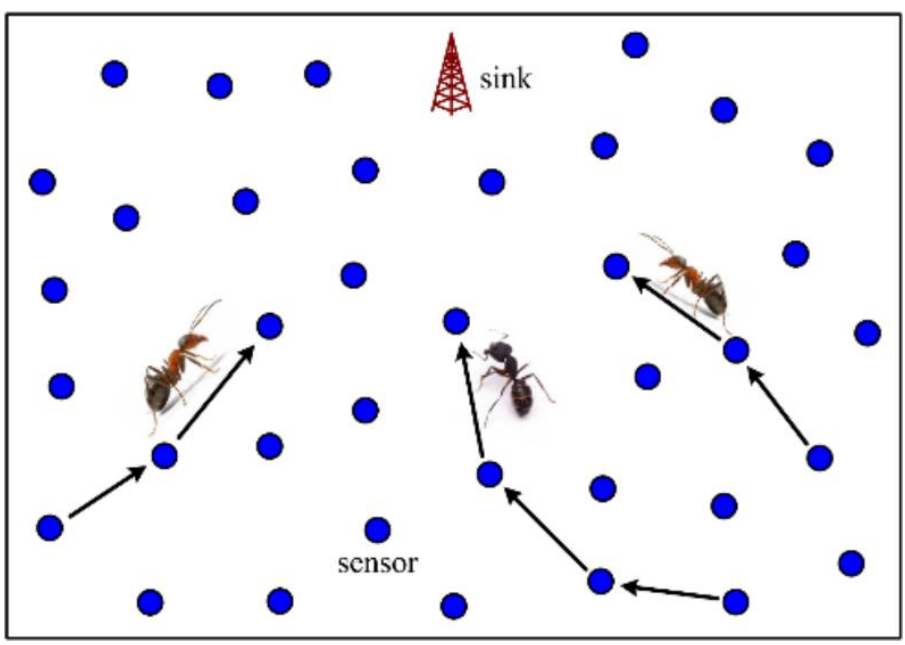

FIGURE 1: Path selection of ACO-based routing.

This proposed method discusses the applications, structures, challenges, and issues we face in designing WSNs. Topology control mechanisms focus more on building a reliable and energy efficient network topology. While sensor cover topology demonstrates network monitoring capability, connection topology should remain as a requirement for the successful delivery of information including queries, data collected, and control messages.

The rest of the paper is organized as follows. In Section2, Research Literature for this study. Section3, Classification of ACO are explained. Proposed Algorithm in Section4. Simulation conditions illustrated figures are given in section5. In section6 explain the investigated amount of network coverage and conclusion of the study is in the last section.

\section{Research literature}

$\mathrm{R} \& \mathrm{M}$ is a distributed topology control protocol based on heterogeneous location-based control protocols. The R\&M protocol creates a topology for all-in-one communications. This type of connection is suitable for networks that have one node as the central node and the other nodes must send their packets to that node. This traffic pattern is a well-known example of sensor networks that need to collect packets of network nodes in one node. How to build a topology with this protocol is that each node sends a Hello message to its neighbors? The node puts that node into its neighbor set for receiving a Hello package from each node. When the direct communication cost of a node with one of its neighbors exceeds the indirect communication cost of the node with that neighbor (through an intermediate node) that neighbor is eliminated from the set of node neighbors. Each node calculates its neighbor set. The graph obtained from the relation of each node to the set of its neighbor nodes constitutes the fence graph. Then, using the classical distributed Bellman-Ford shortest path algorithm on the fence graph, the inverse spanning tree is obtaining with the least energy consumption. The resulting tree is the desired topology $[17,18,19,20,21,22,23,24,25]$.

The LMST protocol is also a type of location-based heterogeneous control protocols. In this protocol, each node sends its information to its neighbors. Each node creates a local minimum spanning tree (MST) for its neighbors for received packets. The topology derived from considering all these local MSTs is the desired topology $[26,27,28]$.

CBTC is a distributed topology control protocol based on heterogeneous direction-based protocols. The basic idea in the CBTC protocol is that the node $u$ transmits its packets with the minimum power $\mathrm{p}$ such that there is at least one neighbor in each cone at the angle $\alpha$ and the center of $u$. The graph obtained by considering these relationships is a directed graph that by adding the return edges to any asymmetric link, the resulting graph becomes unidirectional. It has been shown that network connectivity is provided if $3 / \pi 2 \leq \alpha$. Here's how to get the minimum $p$ value ${ }^{[29,30]}$.

The DSO is one of the searching algorithms which imitates transportation behavior like searching and selecting routes for movement by donkeys in the real world. Two modes are established for applying the search behavior and route-selection in the given algorithm Smuggler and Donkeys. In the Smuggler, possible ways are found and the shortest is selected. In the Donkeys mode, many donkey behaviors are used such as Run, Face \& Suicide, and Face \& Support ${ }^{[31,32,33,34,35,36]}$.

\section{Classification of ACO-based Routing in WSNs}

The Ant Colony Optimization-based routing protocols in WSNs are often classified into multiple varieties. during this section, we have a tendency to propose a comprehensive classification of ACO-based routing protocols in WSNs. As shown in Fig. 2, the classification is achieved from totally different points of read and is analyzed as follow ${ }^{[43]}$.

\section{A. OPERATION BEHAVIOR}

The ACO-based routing in WSNs will be classified into routing of energy state management which of transmission distance management, in step with the operation behavior of the routing. the most operation of the previous is to pick out relay nodes with a lot of energy state, however the latter chiefly aims to pick out applicable transmission distance for every node.

\section{B. ULTIMATE OBJECTIVE}

On the premise of the final word objective, the ACO-based routing protocols in WSNs may also be classified into routing of 
period extension which with QoS demand. the final word goal of the previous is to maximize the network time period, whereas the latter aims to supply specific QoS services.

\section{NETWORK TOPOLOGY}

According to the constellation, the ACO-based routing in WSNs includes 2 varieties, Flat routing and hierarchical routing. In DAT routing, every node performs constant task of path search. However, in hierarchical routing, many nodes ar fashioned into a gaggle and path choice is administrated among completely different teams.

\section{TRANSITION PROBABILITY}

According to the transition chance of the ants, the ACO-based routing in WSNs will be divided into routing of secretion and heuristic which with single secretion. within the former, path choice is performed by each secretion path and heuristic info of the trail, whereas path looking is administered solely by secretion path of the trail.

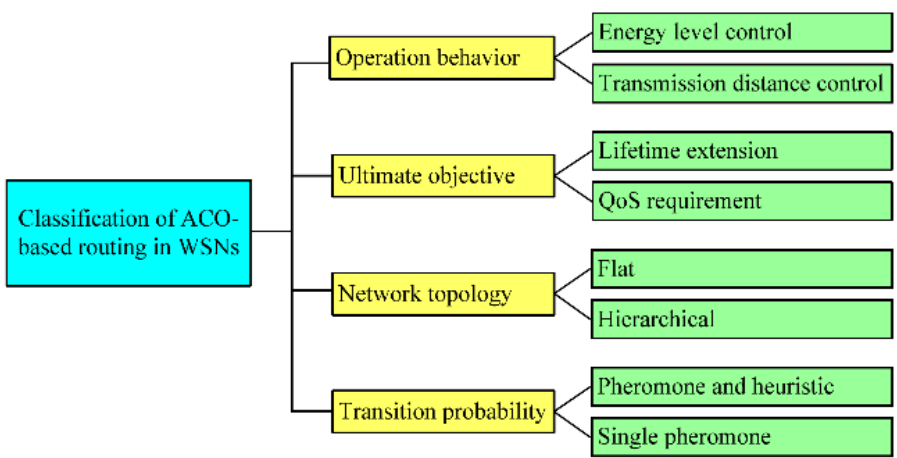

FIGURE 2: Classification of ACO-based routing protocols in WSNs [43].

\section{Proposed Algorithm:}

In the proposed section we classified scheme, plus of monitoring whole topology and maintain nodes connectivity for four phases as we explain one by one from the following:

Phase One:

In the $1^{\text {st }}$ phase of proposed scheme, each node in its cluster declares the amount of energy left over to its current header. The power consumption of our plan is expected to be more balanced and thus increase the lifetime of the network. On the other hand, considering the minimum and optimal number of headers in the network and data aggregation in the header nodes, the cost and energy consumption for sending sensor data by the header nodes is reduced.

score $(\mathrm{i})=(\mathrm{w} 1 *$ number of node $(\mathrm{i}))+(\mathrm{w} 2 *(1 /(1+\operatorname{vardis}(\mathrm{i})))$

$+(\mathrm{w} 3 *(1 /(1+\operatorname{speed}(\mathrm{i})))+(\mathrm{w} 4 * \operatorname{renergy}(\mathrm{i} ;))$

$\mathrm{w} 1=0.5$;

Formula (1).

$\mathrm{w} 2=0.3$

$\mathrm{w} 3=0.1$

$\mathrm{w} 4=0.1$

Phase Two:
Sort and select active (awake) network headers based on the header node privilege then, according to the ratings, we will sort the sector ratings in descending order. The sorting of the sectors is done by ranking and selecting the $\mathrm{K}$ sector of the candidate from the $\mathrm{n}$ sectors in this phase. After selecting each sector, we activate the header (wake up). The header selection is based on the distance parameter of the other nodes from the header node and the residual energy of the header node. The greater the variance of the nodes the greater the node, meaning the sensor nodes are more scattered around the node and therefore the node is not suitable for being high. In point of fact, we want to place a node as a header that will cover more users with the sum of the shorter intervals and be central to the nodes around it. That way we won't have a cover problem, but there is an overlap that will get you to the optimum mode of energy consumption by placing headers in the right places at the right time and removing some inefficient headers. Considering the minimum and optimal number of headers in the network and the data aggregation in the header nodes, the cost and energy consumption for sending sensor data by the header nodes is reduced.

Phase Three:

Integrating Sensor Nodes into Active Headers Using K-Mean and Distance Consideration.

After activating each header, the nodes join to that header as far as possible within the header. In actual fact, we bear the risk of increasing the data transmission distance from node to header so that we can cover all the sensor nodes in the network for data collection with fewer active headers. By reducing the number of headers and, in fact, the number of clusters that are less concentrated in the presence of sensor nodes, we will optimize the installation cost as well as increase the reliability of data transmission. In reality, each become, according to its signal transmitting power, sends a hello packet to its neighbors, requesting to join, announcing its distance to the sensor nodes of the area and the surrounding area.

In the node allocation functions to the header node, if the nearest header to an active node is not set and the number of active headers is also violated, the nearest active header to the node that is within its signal transmission range is selected as the parent of that node (header). Can be.

Finally, the headings are connected to the minimum spanning tree. However, based on the formation of petals between the source and the mobile well, any header on the route to the packet (Located inside the petals) will participate in the routing and deliver the package manually.

Phase Four:

Routing between the header nodes and the well for sending data [31]. Using the ant colony optimization method, the optimal path between the nodes is selected to route the nodes traffic to the well or the main station. So start sending ants to the well at each wellhead to inform the route cost parameters (distance, the quality of each solution, the nodes in that path, and the approximate length of each path to the well). In this way the well will be aware of its distance to each well after some time, and if a better route is delivered to another well at a lower cost through the wellhead node, the table content will be updated so that the well can eventually reach the minimum tree with the closest ones. Between himself and other beings. That is, the tree made by the 
well is being reconstructed over time and shorter alternate paths are added to the tree and the previous ones removed. Subsequently, by sending backward ants, each header will be notified of the selected topology to remove the necessary neighborhood links and other connections. This creates a minimal overlay tree that will cover all the sensor nodes in the cluster in addition to the wellhead and the node. This procedure is performed every $\mathrm{T}$ seconds to generate the optimal topology.

\section{Location in the proposed method}

Node location refers to the location (coordinates) or localization of nodes, since we do not want to depend on the infrastructure and availability of GPS equipment in the nodes, this is done by the RSSI (Received Signal Strength Indicator) method. Because we have divided the simulation environment into sectors, a reference node can be assigned in each sector to send a location signal so that other nodes can derive their distance and spatial coordinates based on the signal strength received.

\section{Simulation conditions}

MATLAB.R2014 software is used to simulate the proposed scheme. The simulation consists of 100 moving nodes located in a $100 \mathrm{~m}$ area with uniform distribution. Each node has a transmission power within 30 meters and an initial energy of 10 units. The number of 100 packet forwarding operations will occur after the topology (via the proposed method) to a specific destination (mobile well). The speed of the nodes varies from 1 to 4 meters per second.

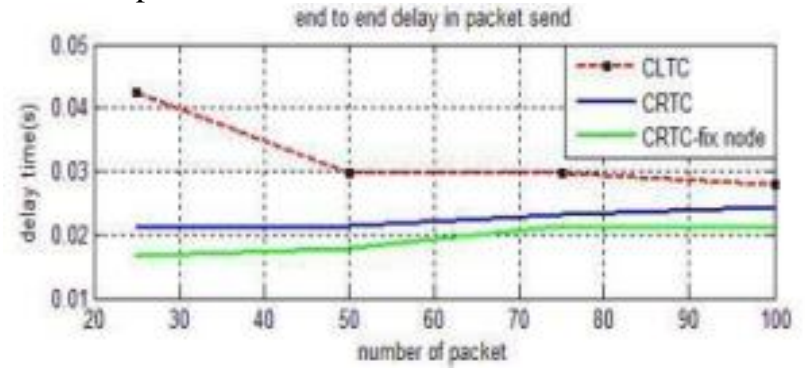

Figure 1: End to end delay in terms of the number of different packets sent

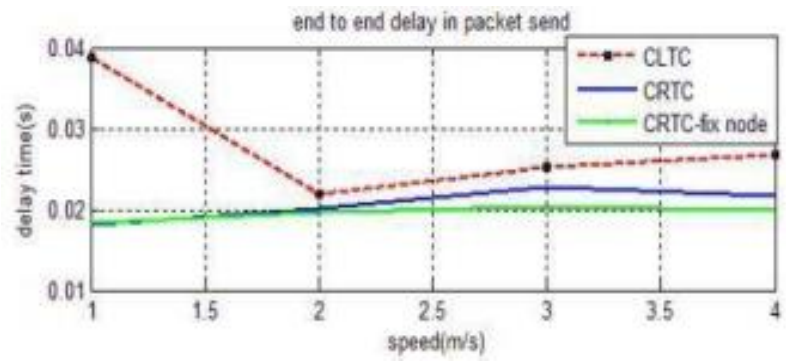

Figure 2: End-to-end delay varies depending on the speed of the node

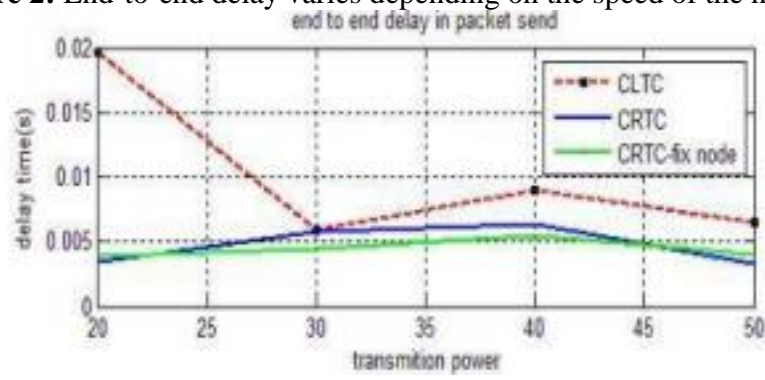

Figure 3: End-to-end delay in terms of different signal transmit power

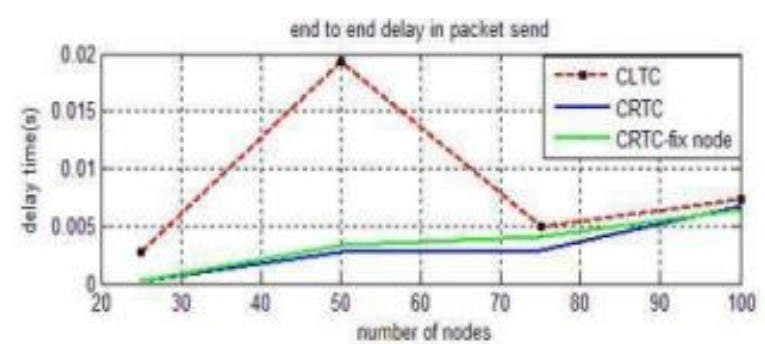

Figure 4: Figure 4. End-to-end delay based on the number of different nodes

The cost is shown in Figures 5 to 8, respectively, by number of different packets, different node movement speed, different signal transmit power, and number of different nodes, respectively.

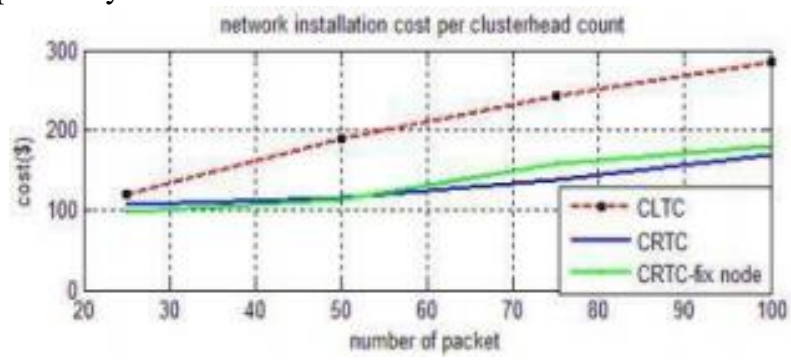

Figure 5: Figure 5. Cost (number of active headers) by number of different packages

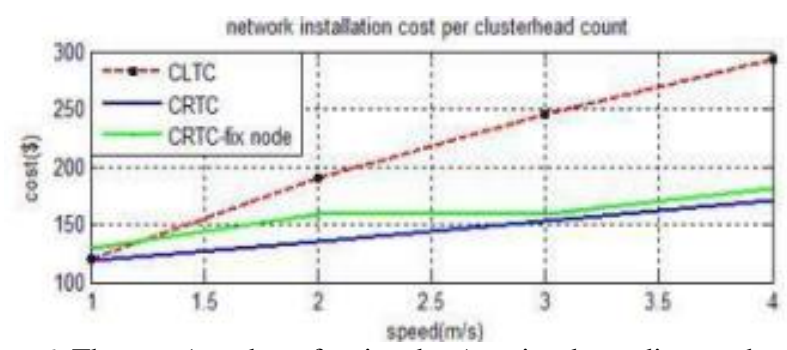

Figure 6: The cost (number of active days) varies depending on the speed of the node

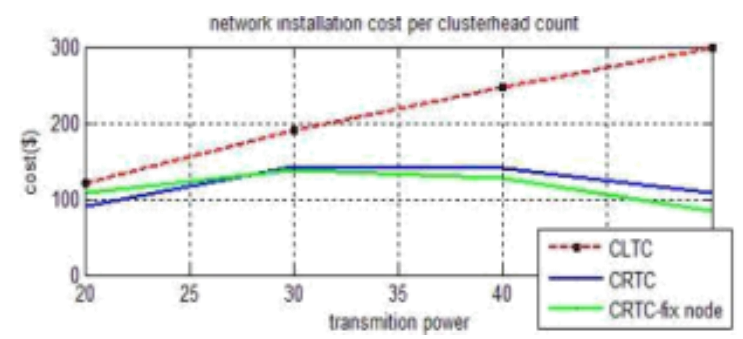

Figure 7: Charge rate (number of active beeps) depending on the signal transmitted power

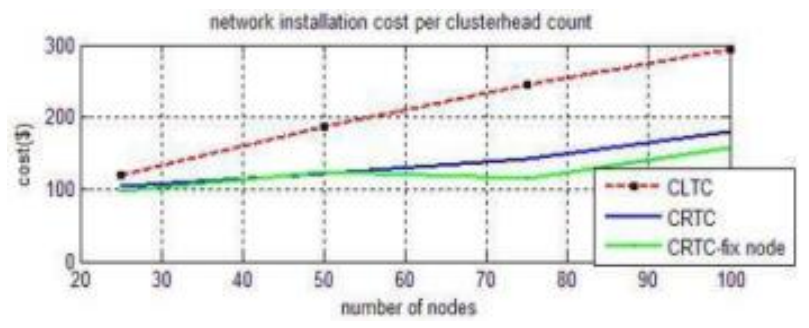

Figure 8: Cost (number of active headers) by number of different nodes

The cost per cover of a sensor node is shown in Figures 9 to 11, respectively, in terms of the number of different packets, the 
different node movement speed, the different signal transmission power, and the number of different nodes.

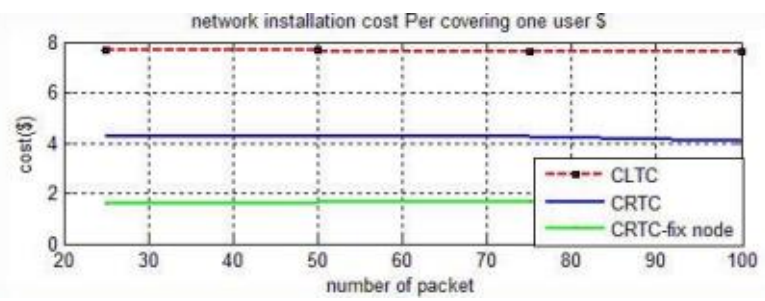

Figure 9: Cost per cover of a sensor node in terms of the number of different

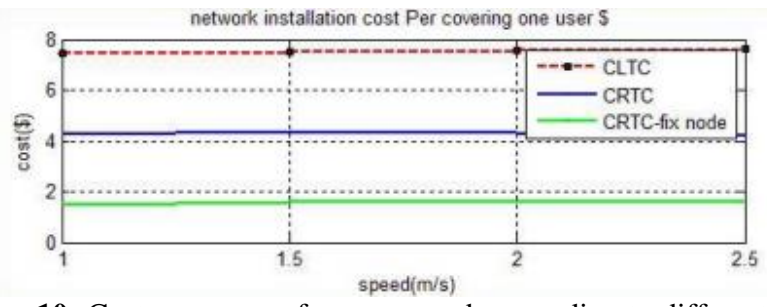

Figure 10: Cost per cover of a sensor node according to different node speed

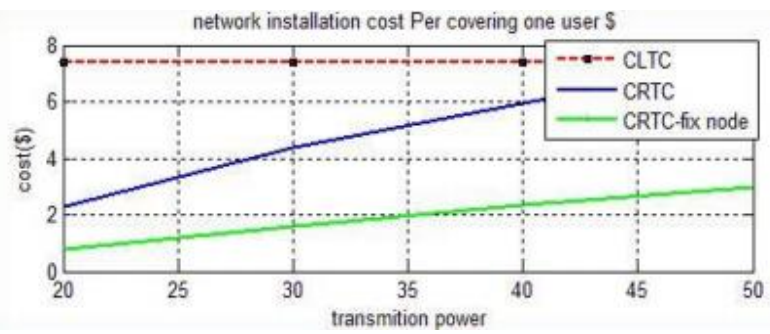

Figure 11: Cost per masking node coverage in terms of different signal transmit power

\section{Network coverage}

To investigate the amount of coverage created in the proposed topology, we simulated the maximum number of clusters $25,20,15,10$ and compared the design performance in terms of coverage. Figures 12 to 15 we find that better coverage is provided in the proposal.

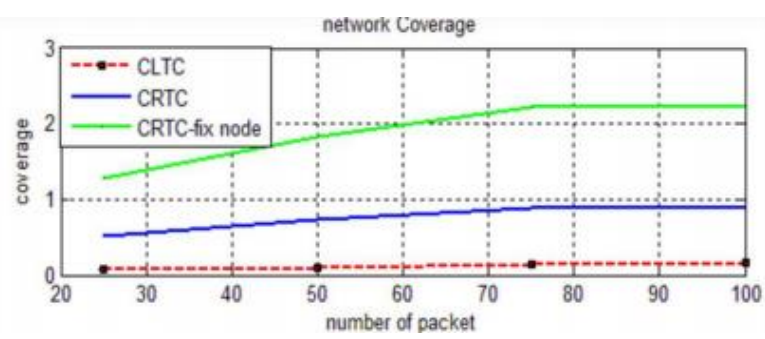

Figure 12: Network coverage by number of different packets

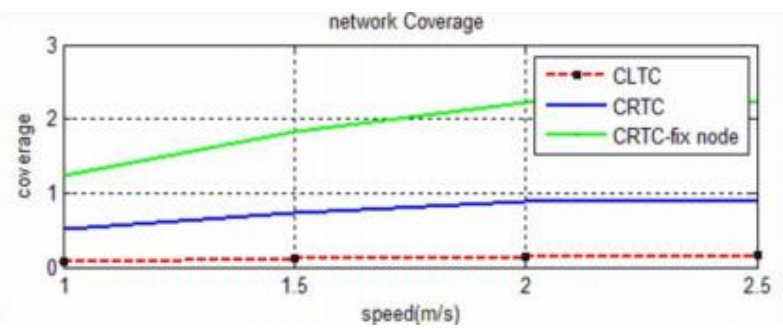

Figure 13: Network coverage according to different node speeds
The network coverage is shown in Figures 12 through 13 in terms of number of different packets, different node movement speed, and different signal transmit power, and number of different nodes, respectively.

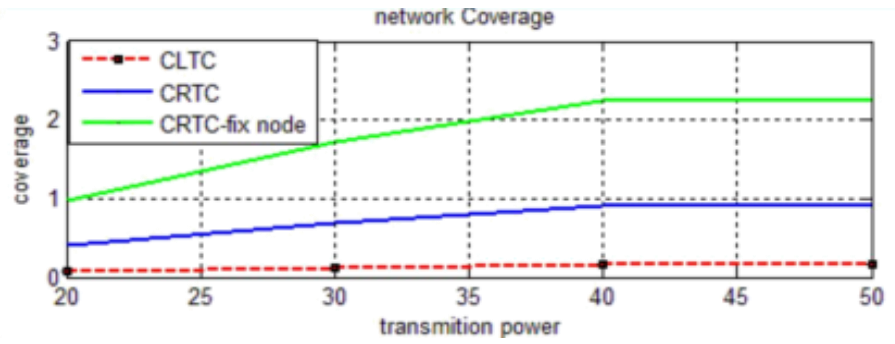

Figure 14: Network coverage in terms of different signal transmission power

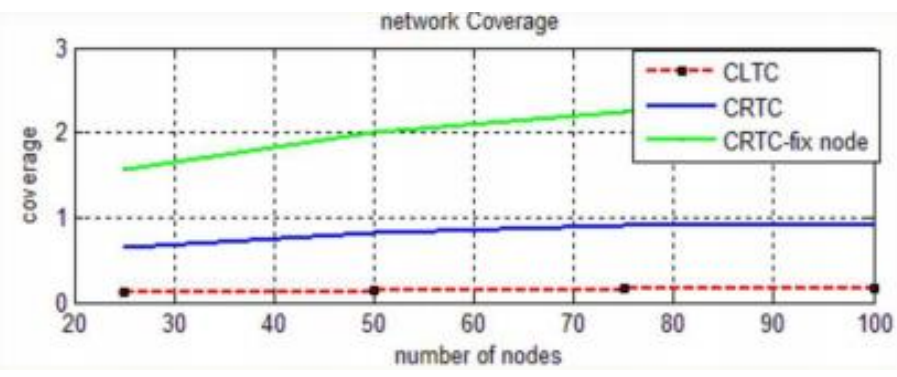

Figure 15: Network coverage by number of different nodes

In the basic design as the criterion, the connection to the nearest header is $\mathrm{K}$-mean and there is no intelligence about the header selection. We may not be able to properly cover all the nodes when we have a limited number of headers. However, if we initially added a header with more nodes around it, we would have achieved better results. To illustrate this concept, we used a visualization of the location of nodes, headers, and how to connect and network together in two basic and proposed designs. As we can see in Figures 16 to 18 , using a limited number of headers, more nodes are covered in the proposed scheme.

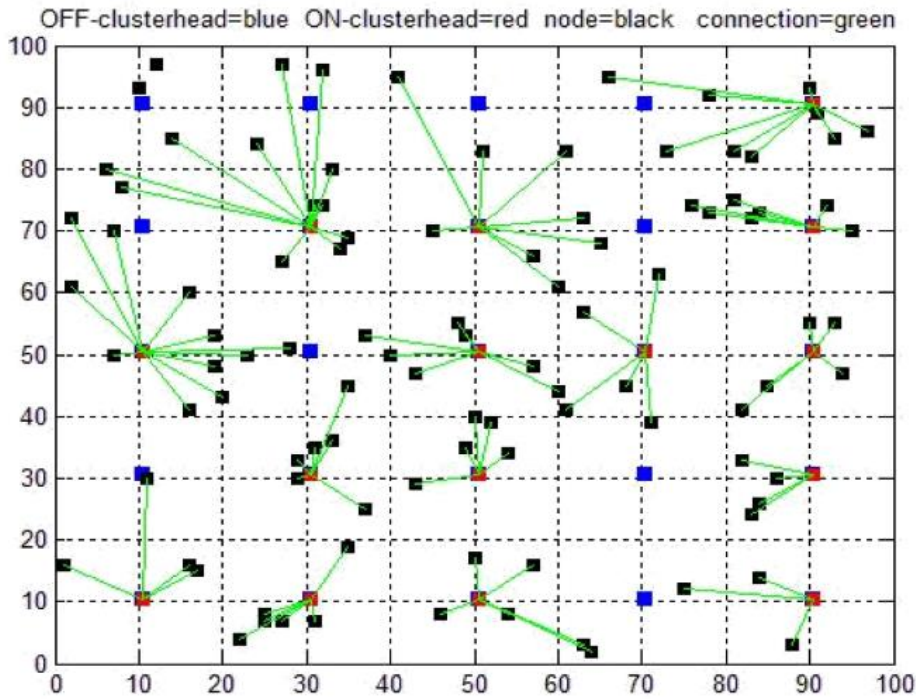

Figure 16: Sensor network topology created by the base design 


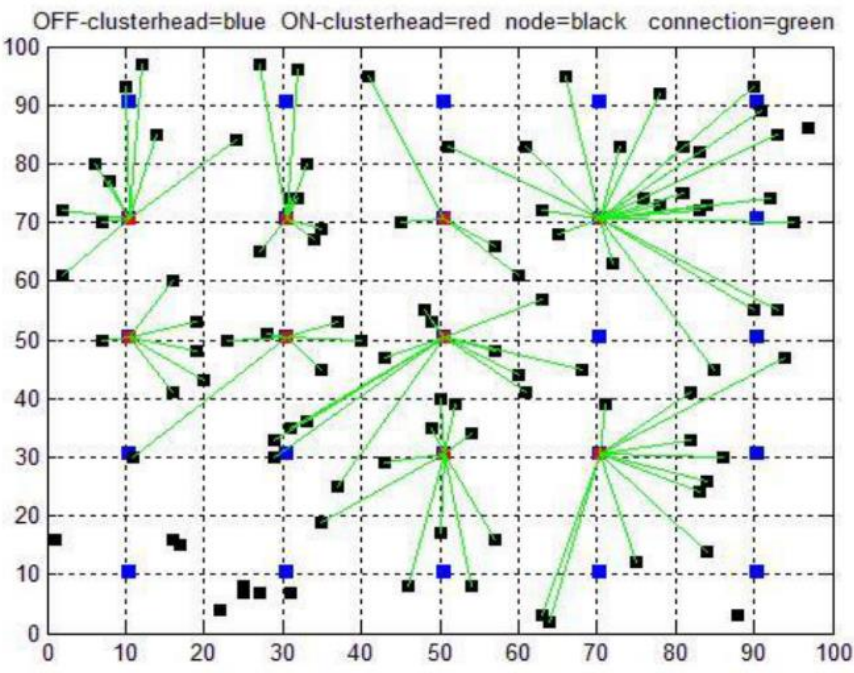

Figure 17: Sensor network topology created by the proposed scheme with moving nodes

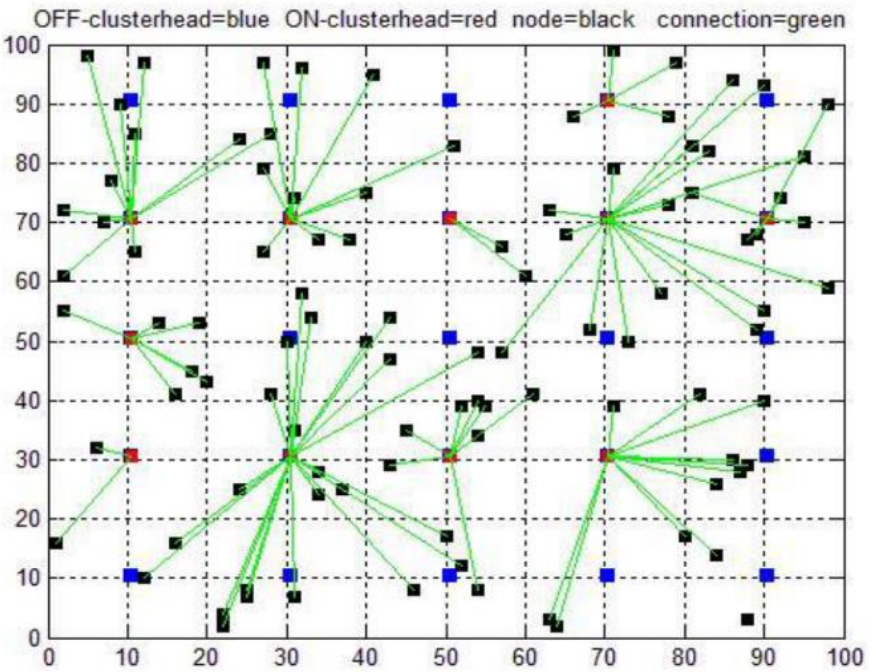

Figure 18: Sensor network topology created by the proposed scheme with fixed nodes

To check the minimum distance between nodes and headers in the designed network topology, we record and compare the sum of the distances the packet sends between the specified source and destination in the formed tree. As we can see in Figure 19, the shorter distances in the proposed scheme (in both fixed and moving nodes) are compared to the baseline.

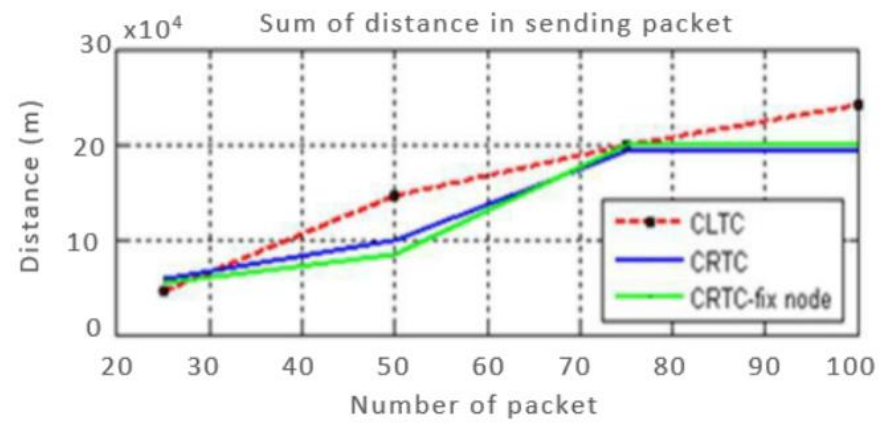

Figure 19: Compares the distance between the header and the node

\section{Conclusion:}

Delivery rates are always higher than the base plan depending on the destinations specified in the proposal. Consequently, the amount of closed-loop loss in the proposal is lower than the base plan. So the reliability of the proposal is higher than the base plan and we have a strong connection in the proposal. The end-to-end delay in the proposal is lower than the base plan. That is, in the proposed scheme, by choosing the appropriate topology with shorter intervals between nodes and header, it will be redirected to the destination with the least amount of time wasted in the optimal and shortest path. The reason for this is the placement of the headers in the center of the cluster, which was the criterion for selecting the headers. In other words, with the type of topology control provided, we will have better coverage (with the maximum number of Maxim headers specified) on deployed sensor nodes. As a result, the packet loss will be lower and there will be less resubmission. To prove the optimality of the topology formed by the implementation of the proposed scheme, we simulated the maximum number of clusters $25,20,15,10$ and compared the design performance in terms of coverage and cost. To do this, we divided the total cost of each case by the number of nodes covered to obtain the cost per node covered. We find that the proposal has lower cost and better coverage. Because in the criterion-based design, the connection to the nearest header is $\mathrm{K}$-mean and there is no intelligence about the header selection. In different implementations, the number of active headers is different, which means that as the network grows larger, the number of active headers cover the network. For example, in the graph of the amount of energy left over by the number of packets sent, the higher the packets, the more network traffic is expected, so the remaining energy is expected to decrease, but as the number of headers manages the network, so less packet loss and less transmissions and energy. Remaining less. We also tested $\mathrm{ACO}$ algorithm and obtained its performance results and examine network lifetime, then we compared the time of death of the first node and the last node in both schemes, and found that network lifetime was increased.

\section{References}

1. G. E. Weir and US Naval History Center, "The American Sound Surveillance System: Using the ocean to hunt Soviet submarines, 1950-1961,” Int. J. Nav. Hist., vol. 5, no. 2, 2006.

2. R. Silva, J. Sa Silva, and F. Boavida, "Mobility in wireless sensor networks - Survey and proposal," Comput. Commun., vol. 52, pp. 1-20, 2014, doi: 10.1016/j.comcom.2014.05.008.

3. A. O. Barznji, T. A. Rashid, and N. K. Al-salihi, "Computer Network Simulation of Firewall and VoIP Performance Monitoring A review of previous research works related to computer network is conducted in," pp. 4-18.

4. V. Shnayder, M. Hempstead, B. R. Chen, G. W. Allen, and M. Welsh, "Simulating the power consumption of large-scale sensor network applications," SenSys'04 - Proc. Second Int. Conf. Embed. Networked Sens. Syst., pp. 188-200, 2004, doi: 10.1145/1031495.1031518.

5. W. Qin, M. Hempstead, and Y. Woodward, "A realistic power consumption model for wireless sensor network devices," 2006 3rd Annu. IEEE Commun. Soc. Sens. Adhoc Commun. Networks, Secon 2006, vol. 1, pp. 286-295, 2006, doi: 10.1109/SAHCN.2006.288433.

6. W. Liu, K. Lu, J. Wang, G. Xing, and L. Huang, "Performance analysis of wireless sensor networks with mobile sinks," IEEE Trans. Veh. Technol., vol. 61, no. 6, pp. 2777-2788, 2012, doi: 10.1109/TVT.2012.2194747. 
7. A. Hasan, T. A. Rashid, B. Ismael, and N. K. AL-Salihi, "Transmission Control Protocol Performance Monitoring for Simulated Wired University Computer Network using OPNET,” UKH J. Sci. Eng., vol. 3, no. 1, pp. 18-28, 2019, doi: 10.25079/ukhjse.v3n1y2019.pp18-28.

8. C. G. Cassandras, W. Tao, and S. Pourazarm, "Optimal routing and energy allocation for lifetime maximization of wireless sensor networks with nonideal batteries," IEEE Trans. Control Netw. Syst., vol. 1, no. 1, pp. 86-98, 2014, doi: 10.1109/TCNS.2014.2304367.

9. G. P. Joshi, S. Y. Nam, and S. W. Kim, Cognitive radio wireless sensor networks: Applications, challenges and research trends, vol. 13, no. 9. 2013.

10. K. Chen and W. Bi, "A new genetic algorithm for community detection using matrix representation method," Phys. A Stat. Mech. its Appl., vol. 535, p. 122259, 2019, doi: 10.1016/j.physa.2019.122259.

11. Q. M. Qadir, T. A. Rashid, N. K. Al-Salihi, B. Ismael, A. A. Kist, and Z. Zhang, "Low power wide area networks: A survey of enabling technologies, applications and interoperability needs," IEEE Access, vol. 6, pp. 77454-77473, 2018, doi: 10.1109/ACCESS.2018.2883151.

12. U. Bilstrup, K. Sjöberg, B. Svensson, and P. A. Wiberg, "Capacity limitations in wireless sensor networks,' IEEE Int. Conf. Emerg. Technol. Fact. Autom. ETFA, vol. 1, no. January, pp. 529-536, 2003, doi: 10.1109/ETFA.2003.1247752.

13. R. Sharma, B. S. Sohi, and N. Mittal, "Hierarchical energy efficient MAC protocol for wireless sensor networks," Int. J. Appl. Eng. Res., vol. 12, no. 24, pp. 14727-14738, 2017.

14. L. M. Rodrigues, C. Montez, G. Budke, F. Vasques, and P. Portugal, "Estimating the lifetime of wireless sensor network nodes through the use of embedded analytical battery models," J. Sens. Actuator Networks, vol. 6, no. 2, 2017, doi 10.3390/jsan6020008.

15. A. H. Pereira and C. B. Margi, "Energy management for wireless sensor networks," SenSys 2012 - Proc. 10th ACM Conf. Embed. Networked Sens. Syst., vol. 1, no. 1, pp. 329-330, 2012, doi: 10.1145/2426656.2426692.

16. H. Yetgin, K. T. K. Cheung, M. El-Hajjar, and L. Hanzo, "A Survey of Network Lifetime Maximization Techniques in Wireless Sensor Networks," IEEE Commun. Surv. Tutorials, vol. 19, no. 2, pp. 828-854, 2017, doi: 10.1109/COMST.2017.2650979.

17. M. Čagalj, J. P. Hubaux, and C. Enz, "Minimum-energy broadcast in all-wireless networks: NP-completeness and distribution issues," Proc. Annu. Int. Conf. Mob. Comput. Networking, MOBICOM, pp. 172-182, 2002.

18. S. Banerjee and A. Misra, "Minimum energy paths for reliable communication in multi-hop wireless networks," Proc. Int. Symp. Mob. Ad Hoc Netw. Comput., pp. 146-156, 2002, doi: 10.1145/513800.513818.

19. X. Y. Li and P. J. Wan, "Constructing minimum energy mobile wireless networks," Proc. 2001 ACM Int. Symp. Mob. Ad Hoc Netw. Comput. MobiHoc 2001, vol. 5, no 4, pp. 283-286, 2001, doi: 10.1145/501449.501460.

20. L. Li and J. Y. Halpern, "Minimum-energy mobile wireless networks revisited," IEEE Int. Conf. Commun., vol. 1, pp. 278-283, 2001, doi: 10.1109/icc.2001.936317.

21. F. Li and I. Nikolaidis, "On Minimum-Energy Broadcasting in All-Wireless Networks,"pp. 193-202, 2001.

22. V. Rodoplu and T. H. Meng, "Minimum energy mobile wireless networks," IEEE J. Sel. Areas Commun., vol. 17, no. 8, pp. 1333-1344, 1999, doi: 10.1109/49.779917.

23. A. Srinivas and E. Modiano, "Finding minimum energy disjoint paths in wireless adhoc networks," Wirel. Networks, vol. 11, no. 4, pp. 401-417, 2005, doi: 10.1007/s11276-005-1765-0.

24. P. J. Wan, G. Călinescu, X. Y. Li, and O. Frieder, "Minimum-energy broadcasting in static Ad Hoc wireless networks," Wirel. Networks, vol. 8, no. 6, pp. 607-617, 2002 doi: 10.1023/A:1020381720601.

25. Y. Wu, P. A. Chou, and S. Y. Kung, "Minimum-energy multicast in mobile ad hoc networks using network coding," IEEE Trans. Commun., vol. 53, no. 11, pp. 1906 1918, 2005, doi: 10.1109/TCOMM.2005.857148
26. D. Das, R. Misra, and A. Raj, "Approximating geographic routing using coverage tree heuristics for wireless network," Wirel. Networks, vol. 21, no. 4, pp. 1109-1118, 2015, doi: 10.1007/s11276-014-0837-4.

27. N. Li, J. C. Hou, and L. Sha, "Design and analysis of an MST-based topology control algorithm," IEEE Trans. Wirel. Commun., vol. 4, no. 3, pp. 1195-1206, 2005, doi: 10.1109/TWC.2005.846971.

28. M. Saravanan and M. Madheswaran, "A hybrid optimized weighted minimum spanning tree for the shortest intrapath selection in wireless sensor network," Math. Probl. Eng., vol. 2014, 2014, doi: 10.1155/2014/713427.

29. S. Hussain and O. Islam, "An energy efficient spanning tree based multi-hop routing in wireless sensor networks," IEEE Wirel. Commun. Netw. Conf. WCNC, pp. 43864391, 2007, doi: 10.1109/WCNC.2007.799.

30. L. Li, J. Y. Halpern, P. Bahl, Y. M. Wang, and R. Wattenhofer, "Analysis of a conebased distributed topology control algorithm for wireless multi-hop networks," Proc. Annu. ACM Symp. Princ. Distrib. Comput., pp. 264-273, 2001, doi 10.1145/383962.384043.

31. L. Li, J. Y. Halpern, P. Bahl, Y. M. Wang, and R. Wattenhofer, "A cone-based distributed topology-control algorithm for wireless multi-hop networks," IEEE/ACM Trans. Netw., vol. 13, no. 1, pp. 147-159, 2005, doi: 10.1109/TNET.2004.842229.

32. A. S. Shamsaldin, T. A. Rashid, R. A. Al-Rashid Agha, N. K. Al-Salihi, and M. Mohammadi, "Donkey and smuggler optimization algorithm: A collaborative working approach to path finding," J. Comput. Des. Eng., vol. 6, no. 4, pp. 562-583, 2019, doi: 10.1016/j.jcde.2019.04.004

33. Hasan, A., Rashid, T. A., Ismael, B., \& Al-Salihi, N. K. (2019). Transmission Control Protocol Performance Monitoring for Simulated Wired University Computer Network using OPNET. UKH Journal of Science and Engineering, 3(1), 18-28.

34. Rashid, T. A., \& Barznji, A. O. (2018). A virtualized computer network for salahaddin university new campus of HTTP services using OPNET simulator. In Online Engineering \& Internet of Things (pp. 731-740). Springer, Cham.

35. Rahman, C. M., \& Rashid, T. A. (2020). A new evolutionary algorithm: Learner performance based behavior algorithm. Egyptian Informatics Journal.

36. M. Dorigo and T. Stützle, Ant Colony Optimization. Cambridge, MA, USA: MIT Press, 2004.

37. E. Bonabeau, M. Dorigo, and G. Theraulaz, "Inspiration for optimization from social insect behaviour," Nature, vol. 406, no. 6791, pp. 39_42, Jul. 2000.

38. J.-W. Lee and J.-J. Lee, “Ant-colony-based scheduling algorithm for energy-ef_cient coverage of WSN," IEEE Sensors J., vol. 12, no. 10, pp. 3036_3046, Oct. 2012.

39. J.-H. Zhong, J. Zhang, "Energy-ef_cient local wake-up scheduling in wireless sensor networks," in Proc. IEEE Congr. Evol. Comput. (CEC), Jun. 2011, pp. 2280_2284.

40. J.-W. Lee, B.-S. Choi, and J.-J. Lee, "Energy-ef_cient coverage of wireless sensor networks using ant colony optimization with three types of pheromones," IEEE Trans. Ind. Informat., vol. 7, no. 3, pp. 419_427, Aug. 2011

41. X.-M. Hu and J.Zhang, "Ant colony optimization for enhancing scheduling reliability in wireless sensor networks," in Proc. IEEE Int. Conf. Syst. Man Cybern. (SMC), Oct. 2012, pp. 785_790.

42. X. Liu, "Sensor deployment of wireless sensor networks based on ant colony optimization with three classes of ant transitions," IEEE Commun. Lett., vol. 16, no. 10,pp. 1604_1607, Oct. 2012.

43. Liu, X. (2017). Routing protocols based on ant colony optimization in wireless sensor networks: a survey. IEEE Access, 5, 26303-26317. 